\title{
First detection of a diamagnetic cavity at comet 67P/Churyumov-Gerasimenko
}

\author{
C. Goetz ${ }^{1}$, C. Koenders ${ }^{1}$, I. Richter ${ }^{1}$, K. Altwegg ${ }^{2}$, J. Burch ${ }^{3}$, C. Carr ${ }^{4}$, E. Cupido ${ }^{4}$, A. Eriksson ${ }^{5}$, C. Güttler ${ }^{6}$,

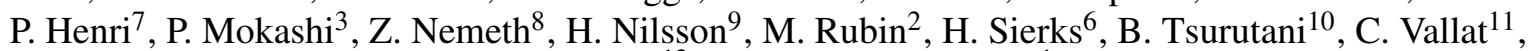 \\ M. Volwerk ${ }^{12}$, and K.-H. Glassmeier ${ }^{1}$ \\ ${ }^{1}$ Institut für Geophysik und extraterrestrische Physik, TU Braunschweig, Mendelssohnstr. 3, 38106 Braunschweig, Germany \\ e-mail: c.goetz@tu-bs.de \\ 2 Physikalisches Institut, University of Bern, Sidlerstrasse 5, 3012 Bern, Switzerland \\ 3 Southwest Research Institute, PO Drawer 28510, San Antonio, TX 78228-0510, USA \\ 4 Space and Atmospheric Physics Group, Imperial College London, Exhibition Road, London SW7 2AZ, UK \\ 5 Swedish Institute of Space Physics, Angström Laboratory, Lägerhyddsvägen 1, 75105 Uppsala, Sweden \\ 6 Max-Planck-Institut für Sonnensystemforschung, Justus-von-Liebig-Weg 3, 37077 Göttingen, Germany \\ 7 Laboratoire de Physique et Chimie de l'Environnement et de l'Espace, UMR 7328 CNRS, Université d'Orléans, 45100 Orléans, \\ France \\ 8 Wigner Research Centre for Physics, Konkoly Thege Miklós út 29-33, 1121 Budapest, Hungary \\ 9 Swedish Institute of Space Physics, PO Box 812, 98128 Kiruna, Sweden \\ 10 Jet Propulsion Laboratory, California Institute of Technology, 4800 Oak Grove Drive, Pasadena, CA 91109, USA \\ 11 European Space Astronomy Centre, 28691 Villanueva de la Canada, Madrid, Spain \\ 12 Space Research Institute, Austrian Academy of Sciences, Schmiedlstr. 6, 8042 Graz, Austria
}

Received 10 November 2015 / Accepted 4 February 2016

\begin{abstract}
Context. The Rosetta magnetometer RPC-MAG has been exploring the plasma environment of comet 67P/Churyumov-Gerasimenko since August 2014. The first months were dominated by low-frequency waves which evolved into more complex features. However, at the end of July 2015, close to perihelion, the magnetometer detected a region that did not contain any magnetic field at all.

Aims. These signatures match the appearance of a diamagnetic cavity as was observed at comet 1P/Halley in 1986. The cavity here is more extended than previously predicted by models and features unusual magnetic field configurations, which need to be explained. Methods. The onboard magnetometer data were analyzed in detail and used to estimate the outgassing rate. A minimum variance analysis was used to determine boundary normals.

Results. Our analysis of the data acquired by the Rosetta Plasma Consortium instrumentation confirms the existence of a diamagnetic cavity. The size is larger than predicted by simulations, however. One possible explanation are instabilities that are propagating along the cavity boundary and possibly a low magnetic pressure in the solar wind. This conclusion is supported by a change in sign of the Sun-pointing component of the magnetic field. Evidence also indicates that the cavity boundary is moving with variable velocities ranging from $230-500 \mathrm{~m} / \mathrm{s}$.
\end{abstract}

Key words. comets: individual: 67P/Churyumov-Gerasimenko - plasmas - magnetic fields - methods: data analysis

\section{Introduction}

The existence of a boundary region between the mass-loaded solar wind and the cometary plasma was first hypothesized by Biermann et al. (1967), who demonstrated with a semi-analytical approach that the solar wind is slowed and redirected when approaching a comet with significant outgassing rates. The incorporation or mass-loading of the heavy cometary ions into the solar wind also leads to the formation of a bow shock on the sunward side of the comet (Szegö et al. 2000; Koenders et al. 2013), then after passing this shock, the solar wind decelerates further and, on the Sun-comet line, eventually stops at the contact surface. Although this purely hydrodynamical approach did not include a treatment of the magnetic field, it was still possible to infer that this so-called contact surface would also affect the interplanetary magnetic field. In a first, simplified model we assume that the magnetic field is frozen into the solar wind flow, and as it decelerates, so does the field, leading to a significant increase in strength. This pile-up then abruptly stops at the contact surface where the magnetic field strength drops to zero because the comet does not have a magnetic field of its own (Auster et al. 2015). This region has been named the "diamagnetic cavity" and is bounded on the outside by an ion composition boundary that the solar wind ions cannot penetrate. This is the cometary ionopause.

The existence of a diamagnetic cavity could not be proven until the Giotto flyby at comet 1P/Halley in 1986. Neubauer et al. (1986) and Neubauer (1988) presented magnetometer data in which a field-free region was detected at a distance of $4760 \mathrm{~km}$ inbound and $3840 \mathrm{~km}$ outbound. This distance was greater than expected for a simple balance between magnetic pressure upstream and dynamic pressure downstream of the boundary. This led Cravens (1987) and Ip \& Axford (1987) to the conclusion that the cavity must be sustained by an ion-neutral friction force. 
The corresponding equation shows the balance of the magnetic pressure and the ion-neutral drag:

$-\frac{\partial}{\partial r}\left(\frac{B^{2}}{2 \mu_{0}}\right)=n_{\mathrm{i}} m_{\mathrm{i}} v_{\mathrm{in}}\left(u_{\mathrm{i}}-u_{\mathrm{n}}\right)$

where $B$ is the magnetic field on the solar wind side, $r$ is the radial distance and $n_{\mathrm{i}}, m_{\mathrm{i}}$ and $u_{\mathrm{i}}$ are number density, mass, and velocity of the cometary ions. $v_{\text {in }}$ is the ion-neutral collision coefficient and $u_{n}$ the neutral gas velocity. Hence, the cavity standoff distance is balanced on one side by the incoming magnetic field and on the other side by the outgassing parameters of the comet. For simple approximations, the ion velocity $u_{\mathrm{i}}$ is often assumed to be zero because the cometary ions and solar wind ions both reach a stagnation point at the cavity boundary. This equation can be used to estimate the distance of the cavity depending on the parameters.

Additionally, Ershkovich \& Mendis (1986) found that the boundary region is susceptible to both Kelvin-Helmholtz and Rayleigh-Taylor instabilities, although the latter requires a very high gravitational force. Later, the evolution of these unstable modes was investigated by Ershkovich \& Flammer (1988) with the conclusion that these modes can indeed convect downstream and reach high amplitudes especially in the case of a weakly outgassing comet (e.g., 21P/Giacobini-Zinner).

Almost three decades after the Giotto flyby at comet 1P/Halley, the Rosetta mission has now afforded a new opportunity to study a cometary plasma environment in situ. The Rosetta spacecraft (Glassmeier et al. 2007a) was launched in 2004 and arrived at comet 67P/ChuryumovGerasimenko (67P) in August 2014. Since its arrival Rosetta scientists have studied the plasma environment of this comet while it approached the Sun and its activity increased. New findings include the detection of low-frequency magnetic field waves (Richter et al. 2015) and heavy ion and solar wind deflection (Nilsson et al. 2015) in the low-activity plasma environment. At a comet-Sun distance of 1.2 AU in July 2015, the Rosetta magnetometer RPC-MAG has detected several signatures of a diamagnetic cavity. The aim of this paper is to provide a first description of the longest duration diamagnetic cavity event and its properties and discuss possible explanations of its unusual features.

\section{Observation}

\subsection{Instrumentation}

All magnetic field data presented here were recorded by the Rosetta Plasma Consortium MAGnetometer (RPC-MAG), a fluxgate magnetometer with a resolution of $39 \mathrm{pT}$ and a range of $\pm 16384 \mathrm{nT}$ (Glassmeier et al. 2007b). RPC-MAG consists of two separate sensors mounted on a boom of $1.5 \mathrm{~m}$ length, one inboard (IB) and one outboard (OB), with a separation of $15 \mathrm{~cm}$. During the interval in question MAG was running in burst mode, meaning an OB sampling frequency of $20 \mathrm{~Hz}$ and an IB sampling frequency of $1 \mathrm{~Hz}$. Because of the small separation of the spacecraft main infrastructure and the magnetometers, the magnetic field measurements are polluted by currents from the spacecraft subsystems. First, the influence of the reaction wheels is profound, but they can easily be filtered out in burst mode because their signature is well known (Glassmeier et al. 2007b). Second, the offset of the magnetic field has an error of $\sim 5 \mathrm{nT}$ in each component that is due to unknown magnetic field sources on the spacecraft as determined from measurements earlier in the mission. Fortunately, the measurements in the cavity can be used to calibrate the data, as it is known from theory that the field inside the cavity must be approximately zero (Biermann et al. 1967).

Unless otherwise indicated, all data are presented in the body-centered solar equatorial frame (CSEQ), where the $x$-axis points toward the Sun, the $z$-axis is the component of the solar north pole that is orthogonal to the $x$-axis, and the $y$-axis completes the right-handed coordinate system. In July 2015, the Rosetta spacecraft was orbiting the comet in the terminator plane, that is, along the day-night line, with a $90^{\circ}$ angle with respect to the Sun-comet line. On July 26, 2015, Rosetta was located $170 \mathrm{~km}$ from the nucleus in the negative $y$ and $z$ quadrant. During the 80-min interval presented here, the spacecraft moved $3 \mathrm{~km}$, which is negligible compared to the dimensions of the plasma region discussed here.

For supplemental information, data from the Rosetta Orbiter Spectrometer for Ion and Neutral Analysis (ROSINA) were used to provide a preliminary estimate of the neutral gas density and gas production rate. Information on outbursts was extracted from OSIRIS images. For further information concerning the ROSINA instrument see Balsiger et al. (2007) and for the OSIRIS camera see Keller et al. (2007).

To estimate the solar wind parameters during this time, we use the model developed by Tao et al. (2005) that is available through the Automated Multi Dataset Analysis (AMDA, amda.cdpp.eu) archiving system. In this model, OMNI data are used to extract input parameters which are the basis for a one-dimensional, spherically symmetric magnetohydrodynamic (MHD) solar wind propagation model.

\subsection{Data}

Figure 1 shows the magnetic field components and the field magnitude on July 26, 2015, corrected for the spacecraft bias field of $6.5 \mathrm{nT}$ by subtracting the mean value of the remaining field in the cavity from each component. The diamagnetic cavity is clearly visible from 15:16 to $15: 41$ as a constant very low magnetic field with almost no wave activity. This lack of fluctuations is remarkable as it is the first time that RPC-MAG has registered no waves at all, which again confirms that this is a diamagnetic cavity. As there is no magnetic field in this region, there can be no magnetic fluctuations. This is the longest interval of cavity measurements up till now, with a duration of $\sim 25 \mathrm{~min}$. It is also preceded by three short dips $(14: 46,14: 54,15: 00)$ that might indicate cavity boundary crossings, but the interval is too short to conclusively prove the presence of a cavity through the lack of wave activity, therefore we focus our studies on the long event.

The average field before the cavity is $(-21.4,0.7,-0.9) \mathrm{nT}$ and afterward $(28.5,2.5,-3.7) \mathrm{nT}$, but the surrounding area is characterized by structures with very high amplitude that are quasi-periodic and asymmetric. RPC-MAG has been observing these kinds of structures intermittently since June 2015 and continues to do so as of October 2015. We discuss these features in a later publication. The structures have a peak-to-peak amplitude of 30-50 nT with a period of 120-170 s. In this interval they are detected mainly in the $B_{x}$ component, except for two cases where the $y$ - and $z$-component also fluctuated heavily. This highly variable field makes it difficult to pinpoint exactly when the spacecraft enters the transition zone around the cavity; the blue shaded areas indicate our estimate of this region. Both crossings were determined on the outside by the fact that the slope of the magnetic field changes at that point in all components. The crossings are $110 \mathrm{~s}$ and $50 \mathrm{~s}$ long. 


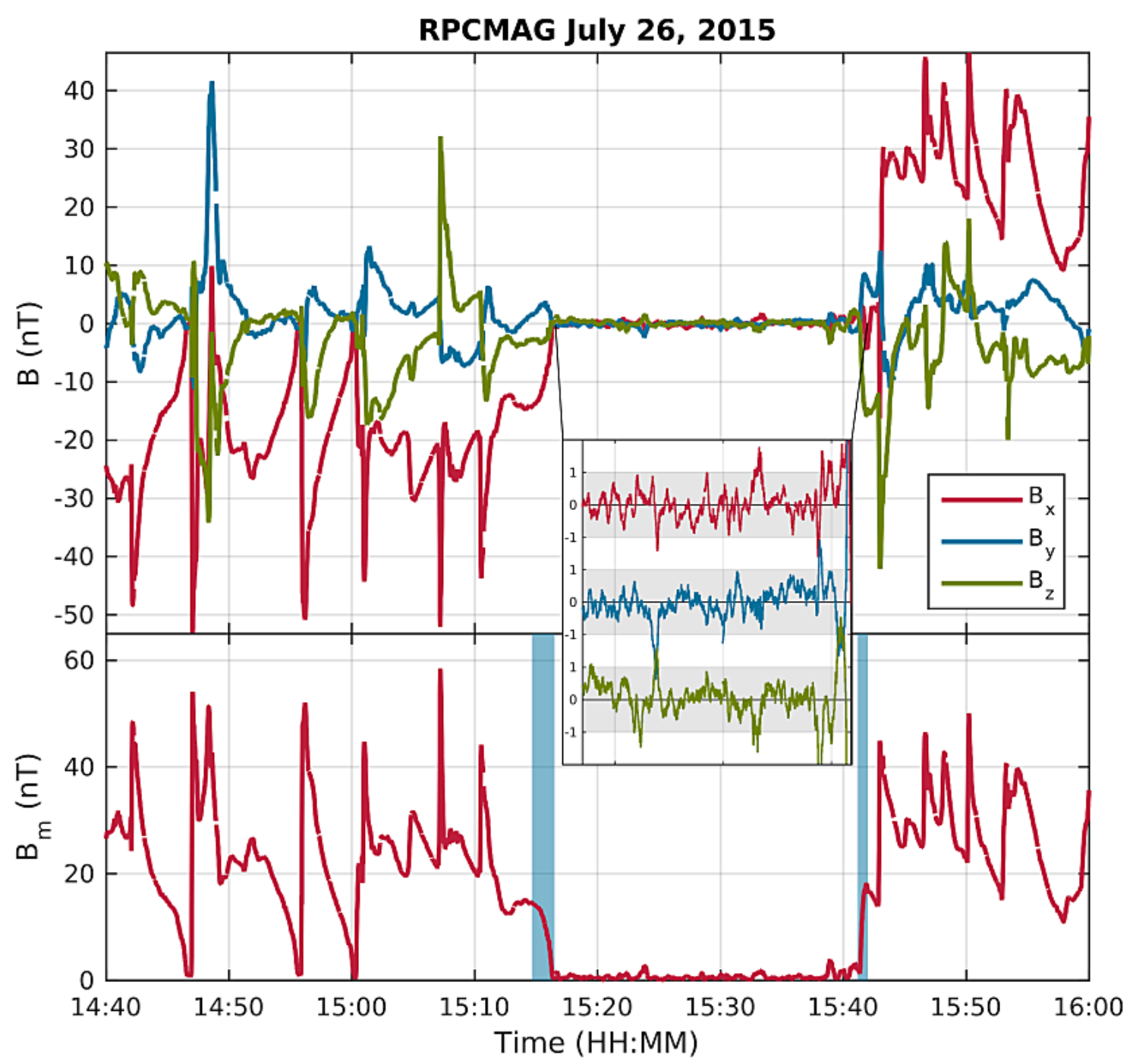

Fig. 1. Magnetic field data on July 26, 2015 from 14:40:00 UT to 16:00:00 UT. The top panel shows the three vector components, which have been corrected by subtracting a constant offset determined by the remaining magnetic field data in the cavity. The magnetic field magnitude shown in the bottom panel has been calculated from the corrected data. The cavity is visible between 15:16:00 UT and 15:41:00 UT, and the shaded areas mark the transition regions. The inset shows a more detailed picture of the three magnetic field components in the diamagnetic cavity.

There were asymmetries between the outbound and inbound diamagnetic cavity traversals. The outbound crossing is a factor of 2.2 shorter than the inbound one. In addition to the faster transition on the outbound leg, there are other notable differences between the two crossings. The transition region is preceded on the outbound path by three smaller quasi-periodic increases starting at 15:38 that are not observed on the inbound leg. During the outbound pass it is notable that $B_{x}$ is a factor of $\sim 2$ smaller than the other two components, before a sudden increase directly after this again makes it the primary component.

A further prominent feature of this cavity crossing is the reversal of the magnetic field in $x$-direction. As seen in Fig. 1, the $x$-component of the magnetic field is negative (pointing away from the Sun) before the cavity encounter and positive (pointing to the Sun) afterward.

There are two small magnetic anomalies in the cavity at $15: 24$ and at 15:33. During the first one, the $y$ - and $z$-components both reach about $2 \mathrm{nT}$, whereas the $x$-component drops from $1 \mathrm{nT}$ to $-1 \mathrm{nT}$, which gives a maximum field strength of $3 \mathrm{nT}$. The second anomaly only has a maximum field strength of $2 \mathrm{nT}$ because only the $x$-component and $y$-component contribute.
Table 1 shows the results of a minimum variance analysis (e.g. Sonnerup \& Cahill 1967) conducted on the ionopause transition, and Fig. 2 displays the magnetic field and boundary normal configuration. The ionopause crossings used for the analysis are $110 \mathrm{~s}$ and $50 \mathrm{~s}$ long. For both intervals the ratio of the eigenvalues is sufficiently high to treat the minimum variance direction as indicative of the boundary normal. During both the inbound and the outbound pass, the boundary normal is quasiperpendicular to the magnetic field, with the main component of the boundary normal in $z$-direction for the former and in $y$-direction for the latter.

\section{Interpretation of the observations}

First, we address the fact that the cavity detected here is significantly farther away from the comet than steady-state simulations (e.g. Koenders et al. 2015) suggest. A possible trigger for the outward motion of the cavity could be a gas and/or dust density increase, which should be detectable by OSIRIS (see Fig. 3). The middle panel approximately coincides with the time when Rosetta entered the cavity, and both the first and second 
Table 1. Results of a minimum variance analysis of the cavity boundary crossings.

\begin{tabular}{cccccc}
\hline \hline Interval & Duration $(\mathrm{s})$ & $n$ & $\theta_{B n}$ & $\lambda_{1}: \lambda_{2}: \lambda_{3}$ & Position $(\mathrm{km})$ \\
\hline 15:14:40-15:16:30 & 110 & $(-0.13,0.53,0.84)$ & $84^{\circ}$ & $1: 11: 268$ & $(-0.1,-99.1,-138.5)$ \\
$15: 41: 10-15: 42: 00$ & 50 & $(-0.29,0.82,0.5)$ & $106^{\circ}$ & $1: 166: 1889$ & $(-0.1,-98.3,-139.0)$ \\
\hline
\end{tabular}

Notes. $\theta_{B n}$ was calculated using average magnetic field values outside the cavity; the angle between the in- and outbound normal is $27^{\circ}$.
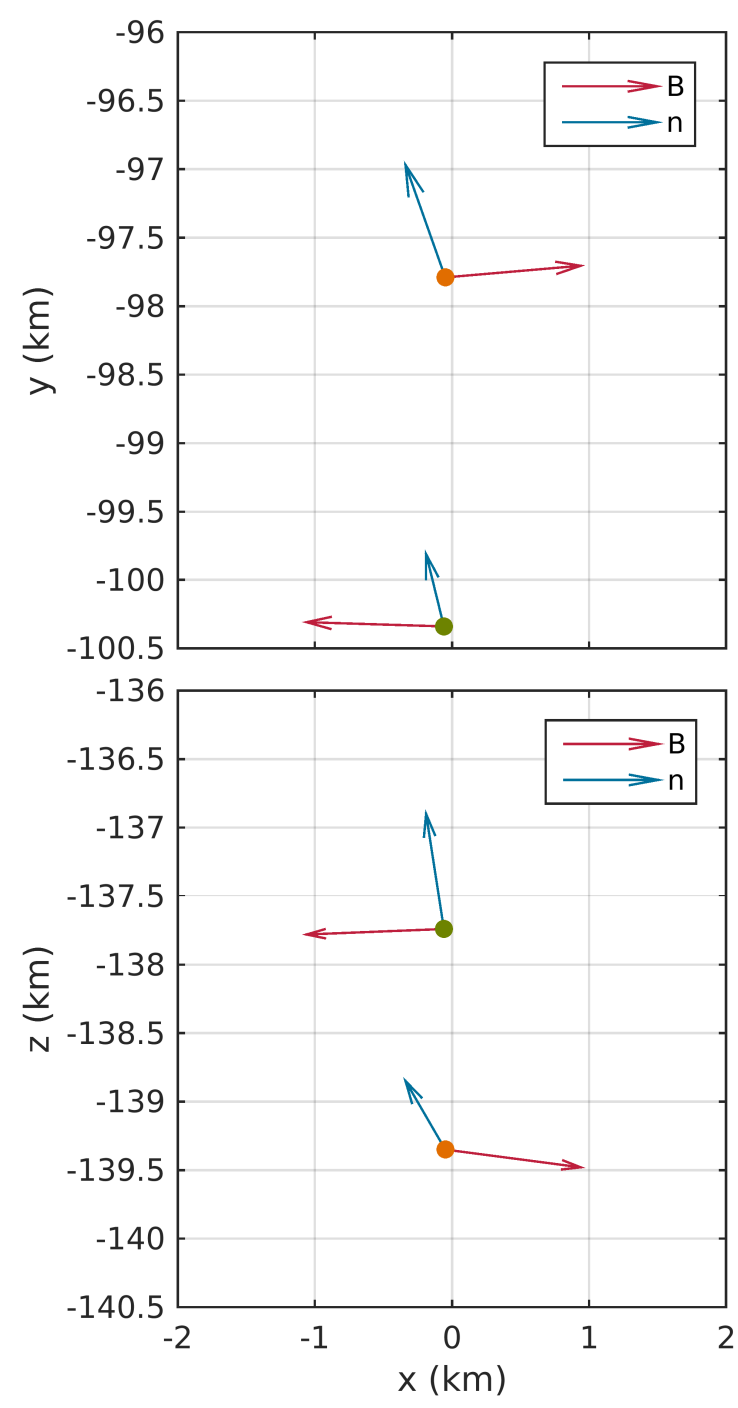

Fig. 2. Normal vector of the ionopause (blue) and averaged magnetic field (red) in the $x-y$ plane (top) and the $x-z$ plane (bottom) in CSEQ coordinates. The green point marks the inbound crossing, the orange point the outbound crossing. We note that the normal vector only indicates the direction and not the orientation of the boundary normal.

image show similar levels of activity, without any remarkable enhancements. The third image is provided for reference, indicating that the activity has slightly increased about an hour after Rosetta leaves the cavity, but this is within the typical range of diurnal variations. This leads us to conclude that the gas production rate is most likely stable during the interval in question. Another possibility is a generally elevated gas production rate and thereby a higher neutral gas density. According to Cravens (1987), the cavity boundary distance is proportional to $Q^{\frac{3}{4}}$ for a steady-state solution with isotropic outgassing. If we assume this to be true and use a reference value of $25 \mathrm{~km}$ for the stand-off distance at a gas production rate of $Q=5 \times 10^{27} \mathrm{~s}^{-1}$ (Koenders et al. 2015), the gas production rate that is required to push out the cavity to a distance of $170 \mathrm{~km}$ can be estimated. To do this, the position of Rosetta during the measurements needs to be considered as well, as the spacecraft was at a $90^{\circ}$ angle from the subsolar point and the cavity does extend farther at these positions. Previously, the cavity has been approximated by fitting a paraboloid to the measurements (e.g. Neubauer 1987, 1988), which implies that the ionopause is farther from the comet at the terminator. Simulations suggest that the cavity boundary distance increases by a factor of 1.5 at these latitudes. With this in mind, the gas production rate for a steady state should be about $3 \times 10^{28} \mathrm{~s}^{-1}$ to achieve the extended cavity we report here.

Second, the gas production rate can also be estimated from the ROSINA neutral gas densities using the Haser model (Haser 1957):

$Q=4 \pi u_{\mathrm{ng}} r^{2} n_{\mathrm{ng}} \exp \left(\frac{v r}{u_{\mathrm{ng}}}\right)$,

where the exponential term can be neglected for the cometocentric distance in question here, because the ionization constant $v$ is about $10^{-7} \mathrm{~s}^{-1}$. A simple estimate for water with a neutral gas velocity of $u_{\mathrm{ng}}=600 \mathrm{~m} / \mathrm{s}$ and values for July 26, 2015, when the neutral gas density $n_{\text {ng }}$ was $2 \times 10^{7} \mathrm{~cm}^{-3}$ and the distance $r$ to the nucleus was $170 \mathrm{~km}$, gives $Q=4 \times 10^{27} \mathrm{~s}^{-1}$. This is one order of magnitude smaller than the estimate above from the cavity crossing at that time. For events at a greater distance, this discrepancy stays roughly the same. It is important to note that this estimate does not take into account coma composition or inhomogeneous outgassing as measured by ROSINA over the course of the mission (Hässig et al. 2015) and therefore provides only a guideline for the outgassing rate. However, it is clear that during the time of the cavity detections, the outgassing rate has to be higher than predicted values. From this first estimate it is unlikely that a change in neutral gas density alone is responsible for the high boundary distance. The right-hand side of Eq. (1) also includes the neutral gas velocity, the ion mass, and the ionization rate. If any of these quantities is higher and not constant, as assumed here, the cavity would also expand.

So far only the right-hand side of Eq. (1) has been investigated, but the left-hand side is also worth studying. The expansion of the cavity may also be triggered by a low magnetic pressure in the incoming solar wind. An estimate of the impinging tangential solar wind magnetic field (Tao et al. 2005) was used to calculate the magnetic pressure in the solar wind around the time of the cavity event. The result together with the magnetic pressure calculated from RPC-MAG data is displayed in Fig. 4. The solar wind dynamic pressure is not shown because it remains unchanged during the interval. The solar wind magnetic pressure as estimated using the Tao et al. (2005) model is significantly lower because Rosetta is situated in the pile-up region, where the solar wind field has been enhanced already. But the solar wind also shows a significant decrease in magnetic pressure around the time when the cavity is observed. The delay time between 

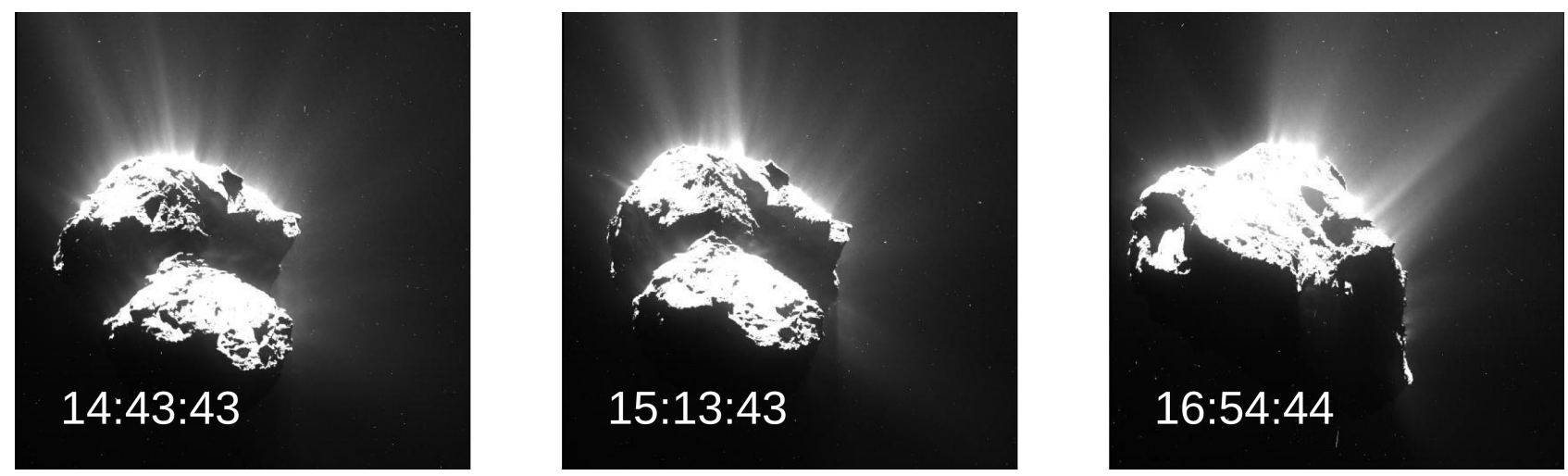

Fig. 3. OSIRIS UV filter images around the time of the cavity detection. The times (UTC) are indicated and all three images were scaled in the same way to visualize the dust coma and make them directly comparable.

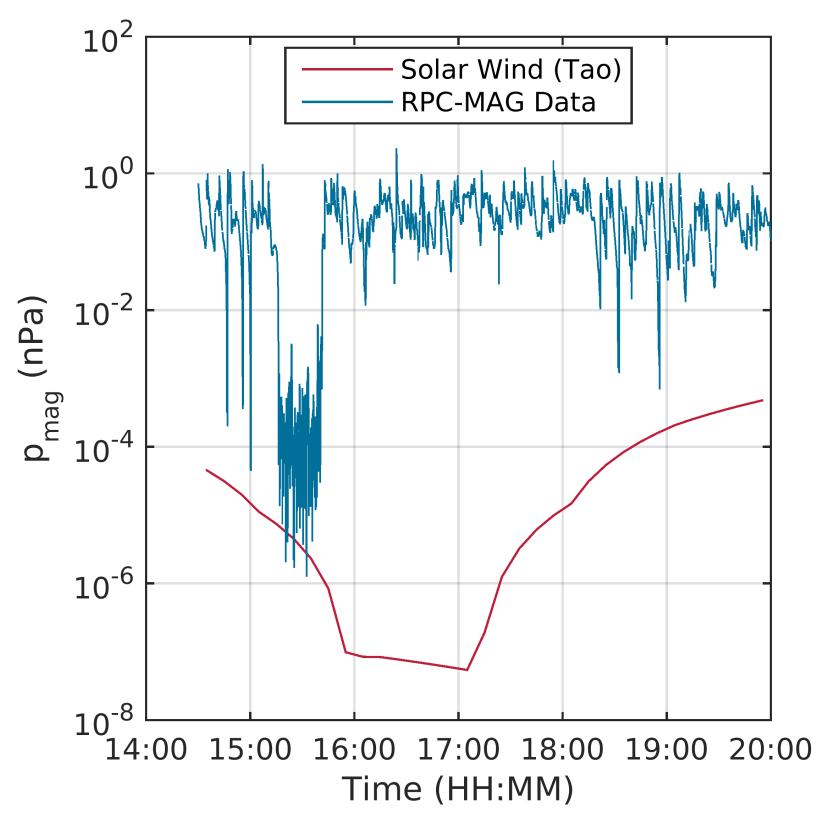

Fig. 4. Solar wind magnetic pressure obtained using the Tao et al. (2005) model and magnetic pressure as measured by RPC-MAG. The lowpressure region in the solar wind lasts from $\sim 16: 00$ to $\sim 17: 00$. We note that to predict the solar wind magnetic field at the comet we used a simple one dimensional MHD model that has large uncertainties.

the estimated solar wind magnetic pressure and the pressure observed by RPC-MAG may be caused by model uncertainties. For a constant radial solar wind magnetic field with constant solar wind dynamic pressure, the maximum magnetic field in the pileup region is lower when the magnetic pressure decreases. This implies that the force created by the magnetic pressure acting on the ion-neutral friction force also decreases. This might explain the outward motion of the cavity. If, by chance, the interplanetary magnetic field reverses in direction during exactly this interval, it would also explain the magnetic field reversal during the crossing

There are two other possible explanations for this situation: either there is an anomalously high density region that is causing the field to loop around, or instabilities propagate along the cavity boundary. We first examine the former possibility. To balance the magnetic pressure, the neutral density needs to be about $10^{9} \mathrm{~cm}^{-3}$, which is three orders of magnitude higher than the ROSINA estimate. We therefore discard this possibility.

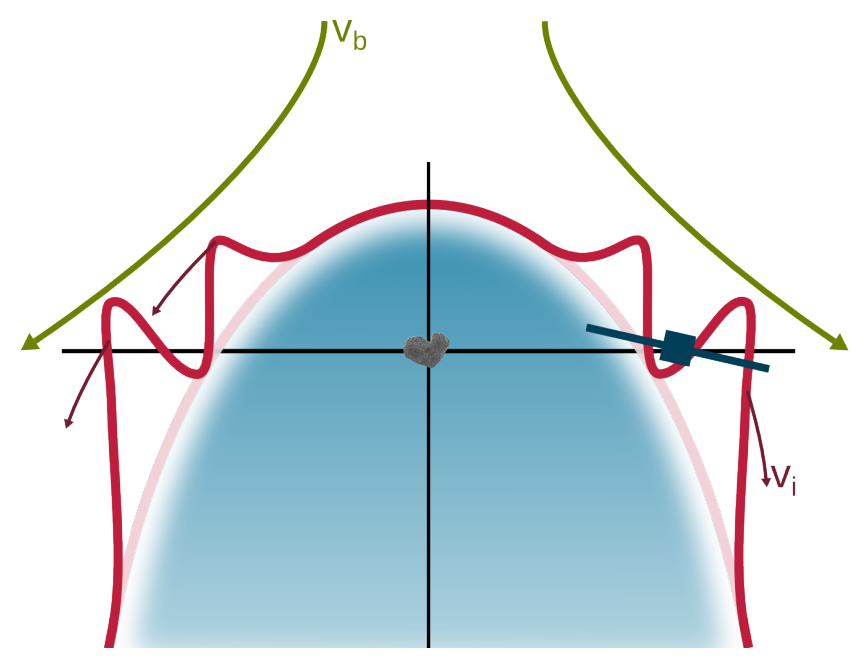

Fig. 5. Sketch of the magnetic field configuration in the frame where the ionopause (red) does not move. The lighter red line indicates the undisturbed cavity boundary. Rosetta's position is indicated in dark blue and the field free region is shaded in blue. $v_{\mathrm{i}}$ indicates the velocity of the instabilities and $v_{\mathrm{b}}$ the velocity of the ion bulk flow (green arrows).

The second option is an instability as investigated by Ershkovich \& Mendis (1986) and Ershkovich \& Flammer (1988). They found that a Kelvin-Helmholtz instability can develop for a sufficiently high velocity shear at the ionopause. The existence of these instabilities was also investigated in MHD (Rubin et al. 2012) and hybrid (Koenders et al. 2015) simulations of the plasma environment of 67P. The former found that these types of instabilities can be triggered by asymmetric outgassing. The latter even confirmed the existence of KelvinHelmholtz instabilities for homogeneous outgassing conditions. In both simulations "pockets" free of magnetic field propagate downstream and significantly alter the magnetic field structure. In the most extreme case this causes a field configuration similar to the one measured by Rosetta during this event; this is illustrated in Fig. 5. The instabilities that are triggered, for example, by a low solar wind magnetic field or an anomalously high gas density, propagate tailward along the cavity boundary and move over Rosetta's position. This way, the magnetic field changes direction, depending on whether it is located between two arms of the instability or entirely outside of the cavity including the unstable region. 
We estimate the lowest bulk flow velocity $v_{\mathrm{b}}$ that is required by the Kelvin-Helmholtz instability criterion. As detailed in Ershkovich \& Mendis (1986), it is possible to solve the Maxwell and MHD equations including the ion-neutral friction and gravity contributions for a dispersion relation that describes both the Rayleigh-Taylor and Kelvin-Helmholtz instability. Then the imaginary part of the dispersion relation (Ershkovich \& Mendis 1986, Eq. (27)) can be used to determine the lowest flow velocity necessary to sustain the instabilities. For comet 67P, the gravity terms contribute little, therefore the Rayleigh-Taylor instability may be neglected. Under the assumption that $v_{\mathrm{b}} \| B$, both orthogonal to the boundary normal and that the plasma number density and ionization coefficient do not change over the ionopause, the remaining instability criterion reduces to

$v_{\mathrm{b}}>\sqrt{2} v_{A}=\sqrt{2 \frac{B^{2}}{\mu_{0} \rho}}$

with $\rho$ the plasma density at the ionopause and $\mu_{0}$ the vacuum permittivity. For a rough estimate we assume that the number density of water ions is around $6000 \mathrm{~cm}^{-3}$ as measured by RPC, and that the magnetic field is $20 \mathrm{nT}$, resulting in $v_{\mathrm{b}}>2 \mathrm{~km} \mathrm{~s}^{-1}$. As the bulk flow mainly constitutes the accelerated cometary ions in this region, it is possible to reach these velocities around a comet. However, this estimate is based on the assumption that the instability is triggered at the point where Rosetta is measuring. If the instability is triggered elsewhere and then convected downstream, the necessary velocity is determined by the parameters at the point of origin.

A moving instability might also explain the asymmetry of the in- and outbound ionopause. The apparently shorter transition time might indicate boundaries moving with differing velocities as seen in Koenders et al. (2015). The velocities $v_{\mathrm{i}}$ may even be calculated if we assume a constant thickness of the transition region. Neubauer (1988) found that the ionopause at 1P/Halley is approximately $25 \mathrm{~km}$ thick, and both Rubin et al. (2012) and Koenders et al. (2015) have reported similar thicknesses. With the transition times given in Table 1 , we calculate velocities of $227 \mathrm{~m} / \mathrm{s}$ for the inbound ionopause and $500 \mathrm{~m} / \mathrm{s}$ for the outbound ionopause. This estimate neglects the spacecraft motion because it is slower than $1 \mathrm{~m} / \mathrm{s}$ during the crossings. Compared to characteristic velocities in the coma, for instance the neutral gas velocity, this is in the same order of magnitude, but slightly lower. Neubauer (1987) also speculated that the ionopause at Halley might have "ripples", which could be interpreted as a precursor to an instability. They were found by comparing the boundary normal of the inbound and outbound crossings. However, it should be noted that at Halley, the boundary normals were on opposite sides of the cometosphere and thus not directly comparable to this case. But the boundary normals here are always approximately perpendicular to the field, which matches our model quite well.

It remains to examine the field configuration in the cavity. So far we are unable to explain the two small anomalies at 15:24 and 15:33, but we can exclude the possibility that they are caused by spacecraft disturbances because the inboard magnetometer measures exactly the same amplitude of the anomalies. The field variation before Rosetta leaves the cavity is most likely caused by the instability of the boundary itself. Ershkovich \& Flammer (1988) have speculated that at 21P/Giacobini-Zinner, the growing instability can break down the boundary and allow the magnetic field to "seep" into the cavity.

\section{Conclusions}

We reported the first detection of a diamagnetic cavity at comet 67P/Churyumov-Gerasimenko. Although the cavity was detected significantly farther away from the nucleus than predicted by simulations the very low field and lack of wave activity make it easily recognizable as a field-free region. A reversal of the magnetic field direction during the crossing led us to conclude that one possibility to explain the anomalously large cavity is the presence of instabilities propagating along the cavity boundary with a velocity of $230-500 \mathrm{~m} / \mathrm{s}$, with an underlying expansion of the entire cavity due to higher gas densities. This assumption can also explain the difference in time for the two ionopause crossings and the ripples preceding the outbound crossing. Other possible explanations are a low and/or rotating solar wind magnetic field or an anomalous neutral gas and ion background. It remains to be seen which of these explanations is most likely by investigating further cavity measurements.

Acknowledgements. The RPC-MAG and ROSINA data will be made available through the PSA archive of ESA and the PDS archive of NASA. Rosetta is a European Space Agency (ESA) mission with contributions from its member states and the National Aeronautics and Space Administration (NASA). The work on RPC-MAG was financially supported by the German Ministerium für Wirtschaft und Energie and the Deutsches Zentrum für Luft- und Raumfahrt under contract 50QP 1401. The work on ROSINA was funded by the federal state of Bern, the Swiss National Science Foundation, and the ESA PRODEX program. Portions of this research were performed at the Jet Propulsion Laboratory, California Institute of Technology, under contract with NASA. We are indebted to the whole of the Rosetta Mission Team, SGS, and RMOC for their outstanding efforts in making this mission possible. We acknowledge the staff of CDDP and IC for the use of AMDA and the RPC Quicklook database (provided by a collaboration between the Centre de Données de la Physique des Plasmas, supported by CNRS, CNES, Observatoire de Paris and Université Paul Sabatier, Toulouse and Imperial College London, supported by the UK Science and Technology Facilities Council).

\section{References}

Auster, H.-U., Apathy, I., Berghofer, G., et al. 2015, Science, 349, 015102

Balsiger, H., Altwegg, K., Bochsler, P., et al. 2007, Space Sci. Rev., 128, 745

Biermann, L., Brosowski, B., \& Schmidt, H. U. 1967, Sol. Phys., 1, 254

Cravens, T. E. 1987, Adv. Space Res., 7, 147

Ershkovich, A. I., \& Flammer, K. R. 1988, ApJ, 328, 967

Ershkovich, A. I., \& Mendis, D. A. 1986, ApJ, 302, 849

Glassmeier, K.-H., Boehnhardt, H., Koschny, D., Kührt, E., \& Richter, I. 2007a, Space Sci. Rev., 128, 1

Glassmeier, K.-H., Richter, I., Diedrich, A., et al. 2007b, Space Sci. Rev., 128, 649

Haser, L. 1957, Bull. Soc. Roy. Sci. Liège, 43, 740

Hässig, M., Altwegg, K., Balsiger, H., et al. 2015, Science, 347, 276

Ip, W.-H., \& Axford, W. I. 1987, Nature, 325, 418

Keller, H. U., Barbieri, C., Lamy, P., et al. 2007, Space Sci. Rev., 128, 433

Koenders, C., Glassmeier, K.-H., Richter, I., Motschmann, U., \& Rubin, M. 2013, Planet. Space Sci., 87, 85

Koenders, C., Glassmeier, K.-H., Richter, I., Ranocha, H., \& Motschmann, U. 2015, Planet. Space Sci., 105, 101

Neubauer, F. M. 1987, A\&A, 187, 73

Neubauer, F. M. 1988, J. Geophys. Res., 93, 7272

Neubauer, F. M., Glassmeier, K. H., Pohl, M., et al. 1986, Nature, 321, 352

Nilsson, H., Stenberg Wieser, G., Behar, E., et al. 2015, Science, 347, 0571

Richter, I., Koenders, C., Auster, H.-U., et al. 2015, Annales Geophysicae, 33, 1031

Rubin, M., Hansen, K. C., Combi, M. R., et al. 2012, J. Geophys. Res., 117, 6227 Sonnerup, B. U. O., \& Cahill, Jr., L. J. 1967, J. Geophys. Res., 72, 171

Szegö, K., Glassmeier, K.-H., Bingham, R., et al. 2000, Space Sci. Rev., 94, 429

Tao, C., Kataoka, R., Fukunishi, H., Takahashi, Y., \& Yokoyama, T. 2005, J. Geophys. Res., 110, 11208 